\title{
Word N-gram Based Classification for Data Leakage Prevention
}

\author{
Sultan Alneyadi, Elankayer Sithirasenan, Vallipuram Muthukkumarasamy \\ Faculty of Science, Environment, Engineering and Technology \\ Griffith University \\ Gold Coast Campus, Australia \\ sultan.alneyadi2@griffithuni.edu.au, \{e.sithirasenan,v.muthu\}@griffith.edu.au
}

\begin{abstract}
Revealing sensitive data to unauthorised personal is a serious problem to many organizations that can lead to devastating consequences. Traditionally, prevention of data leak was achieved through firewalls, VPNs and IDS, but without any consideration to sensitive data. In recent years new technologies such as the data leakage prevention systems (DLPs) are developed, especially to either identify and protect sensitive data or monitor and detect sensitive data leakage. One of the most popular approaches used in DLPs is content analysis, where the content of exchanged documents, stored data or even network traffic is monitored for sensitive data. Content of documents are analysed using mainly text analysis and text clustering methods. Moreover, text analysis can be performed using methods such as pattern recognition, style variation and $\mathrm{N}$-gram frequency. In this paper we investigate the use of $\mathrm{N}$-grams for data classification purposes. Our method is based on using the $\mathrm{N}$-grams frequency to classify documents in order to detect and prevent leakage of sensitive data. We have studied the effectiveness of $\mathrm{N}$-grams to measure the similarity between regular documents and existing classified documents.
\end{abstract}

Keywords- Data leakage prevention; N-grams; N-gram profiles; matching distance

\section{INTRODUCTION}

Leaking data involves release of sensitive information to an unauthorised entity; which might be a result of a deliberate action or a spontaneous mistake [1]. This leakage can lead to catastrophic ramifications to an organization depending on the amount and the value of leaked data. Recent reports show huge losses in both government and commercial sectors, where about $66 \%$ of information security leaks recorded by InfoWatch [2] occurred in commercial sector and about $34 \%$ of the leaks happened in government sector. Although some of the reported leaks were not significant to some organizations, other leaks cost millions of dollars. Commercial loss may occur when sensitive data such as future projects, trade secrets and customer profiles are leaked to a competitor; while government data leak may involve sensitive data of political relationships with other governments, law enforcement and internal security.

A very famous incident which is related directly to leaking sensitive information was the release of United States diplomatic cables through wikileaks website. The incident consisted of revealing about 250,000 United States diplomatic cables and 400,000 military reports called "Iraq War Logs" through an internal entity using an external hard disk. About 100.000 diplomatic cables were labeled as confidential while 15,000 cables were classified as secret [3]. This incident received high level of attention as the United States of America faced many criticisms from many countries and civil rights organisations. Hence these kinds of incidents may cause major financial loss or severely damage an organization's reputation.

In response to such incidents DLP systems have been developed and enhanced to meet the current threats. DLP systems consist of techniques to prevent the leak process by blocking the leaking channel, limit the use of the leaking channel or identify sensitive data and prevent it from leaking outside the secure environment. According to Rich Mogull, current DLP systems are defined as "systems that work under centralised policy to identify, monitor and protect sensitive data in different states through deep content analysis" [4]. Generally data can be found in three states: in transit, in use and at rest. Figure 1 shows different data states.

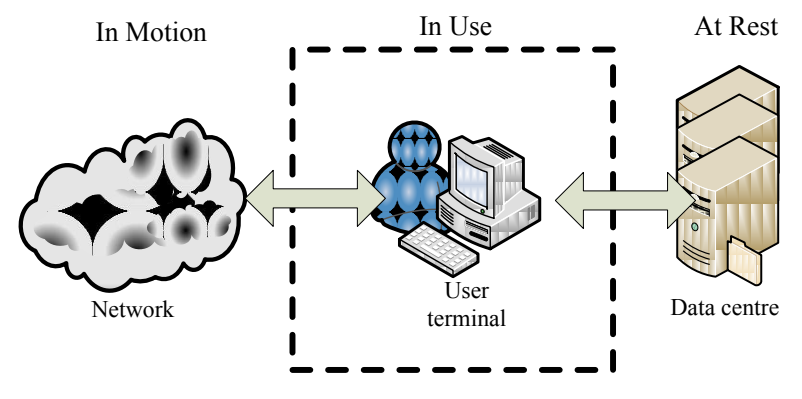

Figure 1. Different data states.

Many DLP systems use text analysis in order to detect sensitive documents. $\mathrm{N}$-grams are widely used in many text analysis tools and in our research we are using word Ngrams to classify documents in order to prevent data leak. The significance of this research comes from the ability to relate unclassified documents to predefined categories instead of actual sensitive documents. Moreover, most of the DLP methods, that uses exact or partial documents detection [5] [6], suffer from serious limitations, such as the susceptibility of hash functions giving different value even with a small change on the original text. This limitation can 
cause sensitive data to be leaked to unauthorized personal without detection. Our research is intended to study the proposed method of using $\mathrm{N}$-gram based classification for data leakage prevention. We are aiming to accomplish three main tasks. First, we are aiming to study the effectiveness of using $\mathrm{N}$-gram based classification for data leakage prevention. This will be done by testing a number of documents against predefined categories, and finding the overall correct classification rate. Second, we are aiming to define an optimum $\mathrm{N}$-gram size -one or two words- along with an optimum category profile size. This will help us to minimize the number of tests and accelerate the classification process. Our third aim is to study the effects of modifying documents on the overall detection.

This paper is divided as follows: Section 2 discusses related work. Section 3 outlines the N-gram classification methodology. Section 4 lists all experiments carried out in our research. Section 5 analyses the findings from the previous section. Section 6 concludes this paper and proposes some future directions.

\section{RELATED WORK}

There are several studies that use $\mathrm{N}$-grams to either categorise documents or detect text variations. For example, Cavnar, W.B. Trenkle, J.M. in [7] studied about the use of $\mathrm{N}$-grams in categorising documents. Their approach achieved high level of accuracy in identifying the language used in the document. It also showed a reasonable accuracy in categorising documents based on their topic. The idea is to break each word in the document into small character Ngrams, and then rearrange them based on the $\mathrm{N}$-gram frequency to create an $\mathrm{N}$-gram profile. Then the created $\mathrm{N}$ gram profile should be compared with existing category $\mathrm{N}$ gram profiles. The document should be classified under the category with the smallest distance measure. This distance is calculated by adding up all the "out of place" values for each N-gram. An example of calculating the overall distance between the category profile and the document profile is shown in figure 2 .

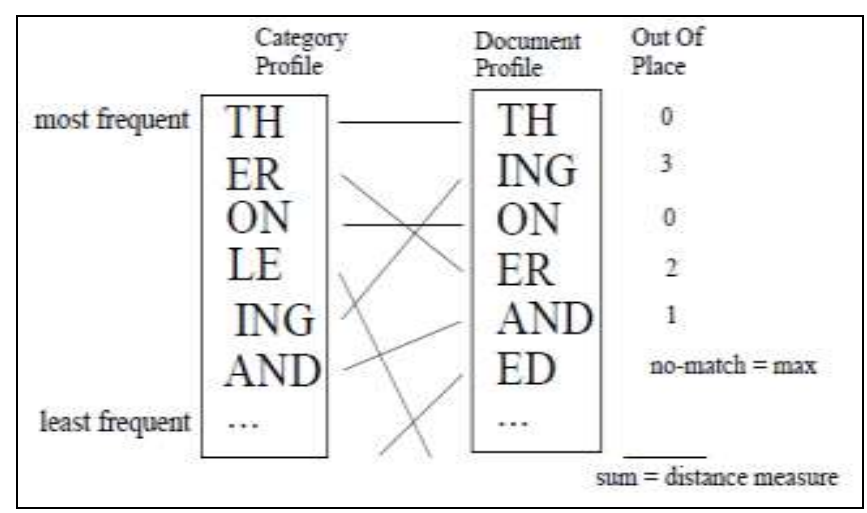

Figure 2. Out of place distance calculation.[7]
The method used in this approach is based on Zipf's law or Zipf's distribution [8]. This law suggests that there is a relation between the number of occurrences (frequency) of a specific word in a natural language -such as English- with its importance (Rank). In other words the bigger the frequency the higher the rank. Use of N-grams also showed a level of accuracy in classifying complex languages like Arabic and Chinese [9] [10] .

Moreover, N-gram classification was used on a dedicated data leakage prevention system. Sokolova, M. et al. [11] presented a system to detect sensitive data in heterogeneous text. This method is based on the separation of possible and impossible containers of personal health information. On their first analysis phase they have used an $\mathrm{N}$-gram classification tool to eliminate non-English text. Furthermore, character $\mathrm{N}$-gram profiles were used in detecting style variation in text in order to detect plagiarized passages. Stamatatos, E. [12] presented an intrinsic plagiarism detection method using a specific sliding window that checks character $\mathrm{N}$-grams and reports any variation in style within a document.

\section{WORD N-GRAM CLASSIFICATION}

Our experiments include testing one-word and two-word $\mathrm{N}$-gram sizes and different document sizes. The process of testing a document for sensitive data is shown in figure 3 . The process requires some prerequisites in order to work correctly. Initially we have identified six categories with corresponding topics. These categories can include any topic that we want to classify or protect. For example if category (Antivirus) is denoted as (A), then category (A) should include information about viruses, malwares, signatures etc.

In our experiments we have created the following categories: antivirus (A), data leak prevention (D), encryption (E), firewall $(\mathrm{F})$, intrusion detection (I) and virtual private network (V). These categories come under information security area and hence successful classification of such linked topics should indicate the effectiveness of our method. Moreover, each category should have two essential things, a classification level and an N-gram profile with a specific N-gram size. The classification levels may include commonly used levels such as: top-secret, secret, and confidential [13]. Since assigning a classification level is totally dependent on the situation, we will not study this problem in this paper. We will mainly discuss the classification process of documents.

After identifying the prerequisites we started testing documents by specifying the $\mathrm{N}$-gram size (one or two words) so we can have similar size to the category profile. Then we sorted the created N-grams by frequency to start the distance measure phase. The document $\mathrm{N}$-gram profiles 
were tested against multiple categories to select the smallest distance. We took into account some factors like the Xcounts (number of unidentified $\mathrm{N}$-grams) and the document size for the analysis phase. Finally, a category was selected after identifying the smallest distance.

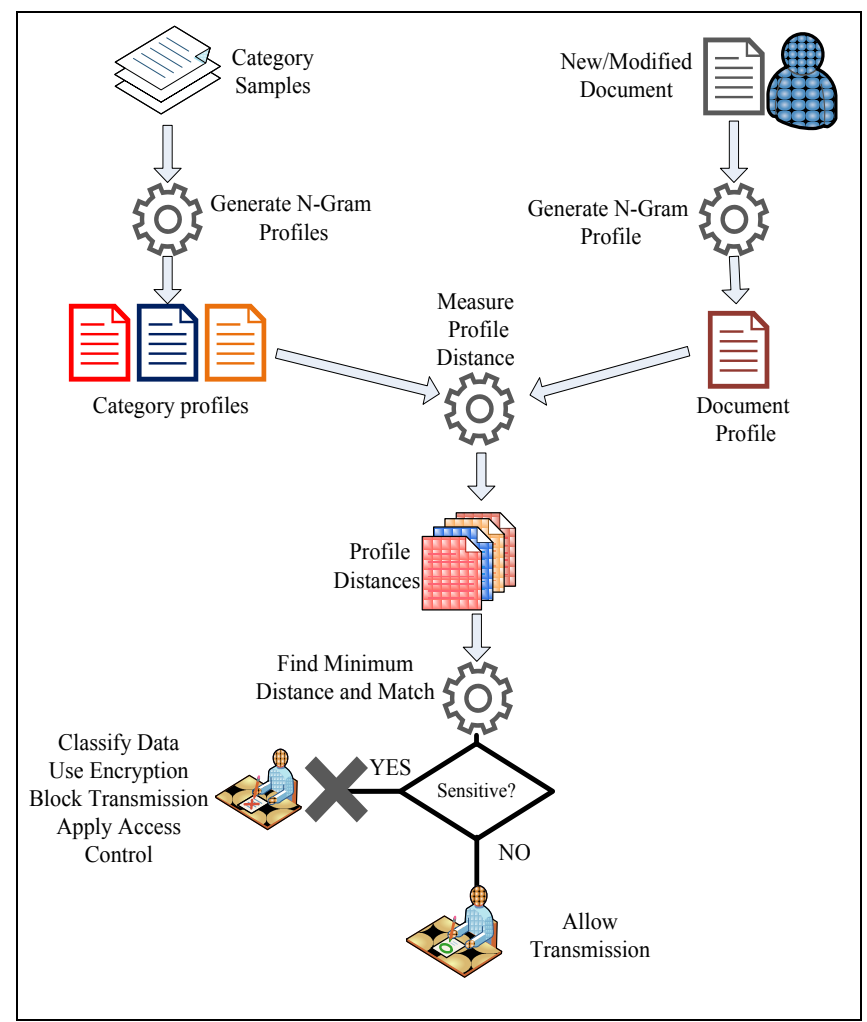

Figure 3. Word N-gram classification process.

\section{A. N-gram Creation}

We have used kfNgram tool [14] to create N-gram profiles. KfNgram tool is a stand-alone free software used for linguistic research. This tool has the ability to create Ngrams from text and HTML source files. The N-grams created by the tool can be (Word or Character) N-grams. For example, if we have the sentence: "the sky is blue", we can create four word-grams of one word such as: the, sky, is, blue. Or we can create three word-grams of two words such as: the sky, sky is, is blue. In this research we have tested both cases and an optimal N-gram size was selected.

\section{B. Distance Calculation}

We developed a MATLAB program to calculate the distance between the document and the category profiles. Initially we started this task by creating a simple code to calculate the N-gram "out of place" distance and the Xcounts. The program uses two sets of strings, one representing the document and the other representing the category. Each string had 7 words representing $7 \mathrm{~N}$-grams. Each word from the document string was compared with all words from the category string. The "out of place" distance was calculated whenever an N-gram was found in the category string. On the other hand a value of 1 was added to the X-count counter whenever the $\mathrm{N}$-gram was not found. This operation was repeated until all $\mathrm{N}$-grams from the document string were compared with all N-grams from the category string. The same concept was applied to the main MATLAB code, but with the addition of some features such as reading from text files and scanning $\mathrm{N}$-grams for similarities. The code calculates the distance and reports the number of unidentified $\mathrm{N}$-grams (X-counts). The X-count refers to (maximum distance) as in [7], and the overall distance between profiles is calculated as follows:

$(X$-counts $\times$ number of $N$-grams in Doc $)+$ "out of place" distance

\section{EXPERIMENTS}

We collected many articles from various sources such as pcmag.com, scmagazine.com etc. These articles were mainly discussing about the six topics we have selected. Also, we have created the category body from the information available on Wikipedia.org. The category profile can be created from any source as long as the content of the source discuses about the same topic and is large enough to create sufficient N-grams. Furthermore, we have created N-gram profiles for both the categories and the articles after omitting all digits and punctuation. This was done by changing some options in the kfNgram tool.

\section{A. Initial Test}

For the initial test we selected five articles from the topic antivirus (A). These articles had different authors and perspective. Also, the articles were different in terms of the size and the writing style. These articles were tested against all six categories. From the initial tests we could not relate any article from topic (A) with category antivirus (A), because of the big distance measure. Most articles were classified under the category data leakage prevention (D). Wrong classifications happened because we used different category profile sizes. In category (A) we used about 1000 $\mathrm{N}$-grams but in category (D) we were only using $440 \mathrm{~N}$ grams. To overcome such a big distance we have tried to reduce the size of the category profile, by considering $\mathrm{N}$ grams that have been sighted at least three times in the category profile. This step reduced the distance significantly, but all articles from topic (A) were again classified under category (D). Next we reduced the category profile size by considering $\mathrm{N}$-grams that have been sighted four times. Out of the five articles two were classified correctly. This gave us an indication that using category profiles with high frequency $\mathrm{N}$-grams should improve the results. 


\section{B. Defining the Optimum Category Profile Size}

Considering the top $150 \mathrm{~N}$-grams in every category profile, we retested the same articles from the initial test. Since the category profiles are sorted by frequency, we are selecting the top ranked $\mathrm{N}$-grams which are reflecting directly to the topic according to Zipf's law. As a result of this we were able to classify all five articles under category (A).

We have extended our testing scale by testing 10 articles from each category using $150 \mathrm{~N}$-gram as a category profile size. That resulted in correctly classifying 47 articles -out of 60 articles- under the corresponding category. Moreover, to define the optimum category profile size, we have tested different category profile sizes. The category profiles sizes with their correct classification results (out of 60 articles) are listed in table 1.

TABLE 1. NuMBER OF CORRECTLY ClasSIFIED DOCUMENTS For EACH CATEGORY PROFILE SIZE

\begin{tabular}{|c|c|c|c|c|c|}
\hline Profile Size & 50 & 100 & 150 & 200 & 250 \\
\hline Correctly Classified Documents & 53 & 49 & 47 & 45 & 45 \\
\hline
\end{tabular}

This proves that the top most N-grams in the category profile represent the topic more effectively; hence size 50 scored the best. To make sure that size $50 \mathrm{~N}$-grams is the optimum size, we extended our testing scale to include size 25 and $300 \mathrm{~N}$-grams. The overall correct classification did not increase by increasing the category profile size and it remained the same for 200, 250 and 300 scales. On the other hand, reducing the category profile size did not increase the overall correct classification. The $25 \mathrm{~N}$-grams profile size scored lower than size 50 profile, as it was only able to detect 48 articles out of 60 , with an overall correct classification reduction of $8.3 \%$. This was an indication that reducing the category profile size to a very small number will give worse results. This is because some of the top ranked $\mathrm{N}$-grams are common in all categories and this may lead misclassification. Table 2 is shows the overall classification results.

TABLE 2. Overall Classification Results For EACH Profile SIZE

\begin{tabular}{|c|c|c|c|c|c|c|c|}
\cline { 2 - 9 } \multicolumn{1}{c|}{} & \multicolumn{7}{c|}{ Category Profile Size } \\
\hline Categories & $\mathbf{2 5}$ & $\mathbf{5 0}$ & $\mathbf{1 0 0}$ & $\mathbf{1 5 0}$ & $\mathbf{2 0 0}$ & $\mathbf{2 5 0}$ & $\mathbf{3 0 0}$ \\
\hline Antivirus & 8 & 10 & 10 & 10 & 10 & 10 & 10 \\
\hline DLP & 9 & 9 & 7 & 7 & 6 & 5 & 6 \\
\hline IDS & 9 & 9 & 10 & 9 & 9 & 7 & 7 \\
\hline Encryption & 5 & 6 & 6 & 5 & 4 & 7 & 7 \\
\hline Firewall & 8 & 9 & 8 & 9 & 9 & 9 & 7 \\
\hline VPN & 9 & 10 & 8 & 7 & 7 & 7 & 8 \\
\hline TOTAL & $\mathbf{4 8}$ & $\mathbf{5 3}$ & $\mathbf{4 9}$ & $\mathbf{4 7}$ & $\mathbf{4 5}$ & $\mathbf{4 5}$ & $\mathbf{4 5}$ \\
\hline \% & $\mathbf{8 0 . 0 0}$ & $\mathbf{8 8 . 3 3}$ & $\mathbf{8 1 . 6 7}$ & $\mathbf{7 8 . 3 3}$ & $\mathbf{7 5}$ & $\mathbf{7 5}$ & $\mathbf{7 5}$ \\
\hline
\end{tabular}

Also, it should be noted that due to practical reasons we have chosen to step the category profile size by 50 , except in the case of minimum size where we have used $25 \mathrm{~N}$ grams. It may have been better to carry out these experiments in steps of 10 or 5 , but since this granularity will invariably depend on the size of the category profile, we presume it will not have a significant impact on the overall detection rate

\section{Defining the Optimum N-gam Size}

By splitting the previous experiment results into two (one-word and two-word N-grams), we checked the classification performance for each size. In most cases oneword N-gram showed better overall detection. For example, all categories scored higher number of correct classification using one-word $\mathrm{N}$-gram except category (I). The highest correct classification number achieved by one-word $\mathrm{N}$ grams using size 50 category profile was 28 articles out of 30 . While the highest correct classification number achieved by two-word $\mathrm{N}$-grams using size 50 category profile was 26 articles out of 30 . The graph shown in figure 4 is comparing between the detection of one-word and two-word $\mathrm{N}$-grams.

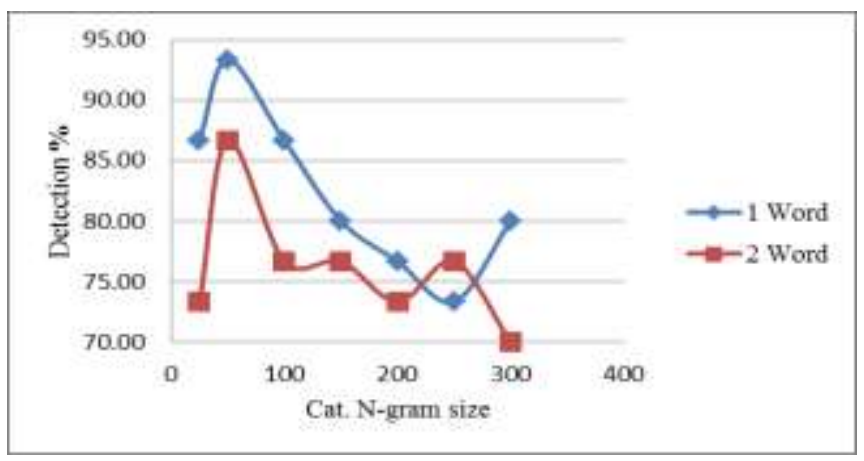

Figure 4. Detection using one-word and two-word N-grams.

From these findings we came to the conclusion that a category profile with 50 one-word $\mathrm{N}$-grams should give the best results.

\section{Overall Classification}

After defining the optimum classification values from the previous test, we tested 180 articles to confirm our findings. All the articles were directly or partially related to the topic, yet the results were encouraging. The results came with an overall of $78.8 \%$ correct classification. Moreover, it was clear that both antivirus (A) and encryption (E) categories scored the highest correct classification of $97 \%$ (29 articles of 30), while DLP (D) category scored only $47 \%$ (14 articles of 30). The reason behind that may be the category profile which contains less relevant $\mathrm{N}$-grams words-. Table 3 shows the overall detection results for each category. 
TABLE 3. OVERALL DETECTION FOR EACH CATEGORY

\begin{tabular}{|c|c|c|c|c|c|c|}
\hline & \multicolumn{6}{|c|}{180 Articles } \\
\hline & $\mathbf{A}$ & D & I & $\mathbf{E}$ & $\mathbf{F}$ & $\mathbf{V}$ \\
\hline Categories & 30 & 30 & 30 & 30 & 30 & 30 \\
\hline Antivirus & 29 & 3 & 2 & 1 & 3 & 0 \\
\hline DLP & 0 & 14 & 0 & 0 & 0 & 0 \\
\hline IDS & 0 & 5 & 26 & 0 & 1 & 1 \\
\hline Encryption & 1 & 6 & 0 & 29 & 2 & 2 \\
\hline Firewall & 0 & 2 & 2 & 0 & 24 & 7 \\
\hline VPN & 0 & 0 & 0 & 0 & 0 & 20 \\
\hline Correct & 29 & 14 & 26 & 29 & 24 & 20 \\
\hline Detection \% & $97 \%$ & $47 \%$ & $87 \%$ & $97 \%$ & $80 \%$ & $67 \%$ \\
\hline Average & $78.8 \%$ & & & & & \\
\hline
\end{tabular}

\section{ANALYSIS}

\section{A. $\quad$-gram Detection Rate}

To have a comprehensive view about our findings we have studied the relation between the document size and the $\mathrm{N}$-gram detection rate. The document size can refer to two things. First, the size of the document's N-gram profile (how many different $\mathrm{N}$-grams are there in the document profile?), second, the size of the document file in kilobytes. For example we can have 10 pages long document which has $30 \mathrm{~kb}$ of data, but contains only one word. In this case we might have a big document in terms of the data size, but we will end up with a small $\mathrm{N}$-gram profile. The relation between the document's N-gram profile size and the $\mathrm{N}$ gram detection rate is shown in figure 5.

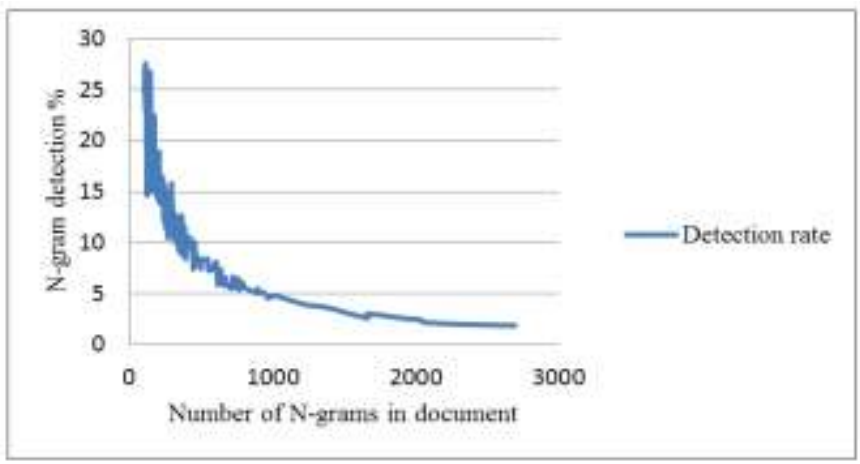

Figure 5. Document size Vs N-gram detection rate.

Since we are using $\mathrm{N}$-gram detection rates from correctly classified document, we can obtain important information from figure 5. The graph is showing a proportional relation between the document's profile size and the N-gram detection rate. For example, the graph shows $5 \%$ detection rate when the document's profile is around $1000 \mathrm{~N}$-grams. This means that around $50 \mathrm{~N}$-gram were detected. Also, the $\mathrm{N}$-gram detection rate was around
$7 \%$ for a document's profile of $500 \mathrm{~N}$-grams. This means that around $35 \mathrm{~N}$-grams were detected. Using the plotted graph we can estimate the number of relevant $\mathrm{N}$-grams in a document. This may help in defining a minimum N-gram detection rate that can be used as a detection measure in the future.

We have calculated the $\mathrm{N}$-gram detection rate using a simple equation that represents the percentage of the identified $\mathrm{N}$-grams. The equation simply says:

$$
\text { Detection rate }=\frac{y \text { counts }}{\text { No.of Ngrams in Doc. }} \times 100
$$

Where: $\mathrm{Y}$-counts $=$ Number of $\mathrm{N}$-grams detected $=($ Number of $\mathrm{N}$-grams in document - $\mathrm{X}$-counts)

\section{B. X-counts}

By plotting the recorded X-counts for each document's test along with the recorded distance measure, we have discovered that the relation between $\mathrm{X}$-counts and the distance measure initially took a linear shape. The initial readings indicate that the increase in $\mathrm{X}$-counts did not meet sharp increase in distance. However after exceeding $500 \mathrm{X}$ counts the distance measure sharply increased. This relation is shown in figure 6 .

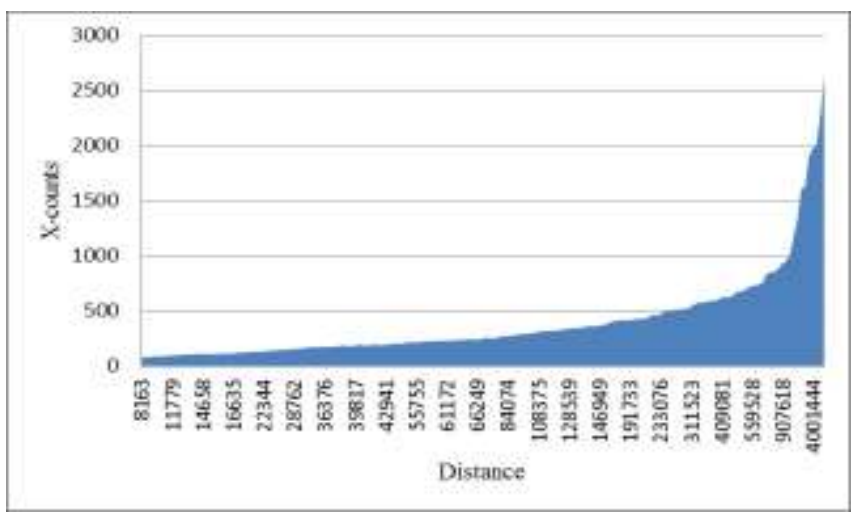

Figure 6. X-counts effect on the overall distance.

Because we are using data from more than 150 correctly classified documents, we can use the graph in figure 6 to approximate distance for documents that belong to one of the categories.

\section{Category Profile Modification}

The experiments carried out on 180 documents resulted in $78.8 \%$ correct classification. Also, the DLP (D) category scored the lowest detection, where only 14 out of 30 documents were classified correctly. To investigate this problem we manually checked the document profiles of the 30 documents for common high ranked N-grams. We came across the following words as most common but not listed in the category profile: Files, leaving, critical, encryption, 
protect, loss, leak, insider, block and disclosed. We added these words to the (D) category profile without removing any existing $\mathrm{N}$-gram. The total number of $\mathrm{N}$-grams in the category profile was 60 . We performed the experiment again for the 30 DLP documents using the new (D) category profile. After modifying the category profile and adding only 10 more $\mathrm{N}$-grams the detection increased from 14 to 25 , percentage wise $47 \%$ to $83 \%$. And the overall correct classification increased from $78.8 \%$ to $85 \%$.

\section{Document Modification}

In this experiment we have tested five articles from each category and we have modified the body of the articles by subtracting and adding paragraphs from various sources. All articles used in this experiment have been already used in the main experiment and were correctly classified. The aim of the experiment is to simulate modification of documents and then classifying the documents. Therefore we have manually tampered the articles' body by deleting some paragraphs and adding some irrelevant paragraphs from different topics such as medicine and business. The modification varied between subtracting $12.1 \%$ to $35.3 \%$ of the original article size, and adding $10.7 \%$ to $112.2 \%$ of the original body. It should be noted that adding irrelevant words to the original document can take any position within the text's body, since all words will be arranged according frequency.

The results showed high level of correct classification of sensitive documents even after modifications. We were able to detect 28 documents of 30 . Only one document from category $(\mathrm{F})$ was classified as $(\mathrm{V})$ and only one document form category (V) was classified as (E). Although it was wrong classification but both correct categories scored the lowest $\mathrm{X}$-counts among other categories. These results give clear indication that $\mathrm{N}$-gram based classification can be effective even with document modification.

\section{E. Precision and Recall}

To evaluate our method we have chosen precision and recall measures in our analysis. In our research as in [9] we define precision as the ratio between correctly classified articles and the number of overall detected articles. Recall is defined as the ratio between correctly classified articles and the number of desired detections. We have studied the precision and recall measures for each category profile size, and from table 4 we can see that size $50 \mathrm{~N}$-gram scored the highest precision and recall among other profile sizes. On the other hand size $250 \mathrm{~N}$-grams scored the lowest precision and recall.
TABLE 4. Average Precision And ReCALl for Each Profile SIZE

\begin{tabular}{|c|c|c|c|c|c|c|c|}
\cline { 2 - 8 } \multicolumn{1}{c|}{} & $\mathbf{2 5}$ & $\mathbf{5 0}$ & $\mathbf{1 0 0}$ & $\mathbf{1 5 0}$ & $\mathbf{2 0 0}$ & $\mathbf{2 5 0}$ & $\mathbf{3 0 0}$ \\
\hline Precision & 0.826 & 0.829 & 0.796 & 0.796 & 0.806 & 0.775 & 0.782 \\
\hline Recall & 0.800 & 0.883 & 0.817 & 0.783 & 0.750 & 0.750 & 0.750 \\
\hline
\end{tabular}

To have a better understanding, we plotted the average of both the precision and recall found in every category profile size testing. Figure 7 illustrates the average of both precision and recall. Size $50 \mathrm{~N}$-gram's scores are represented by the two peaks in the two lines.

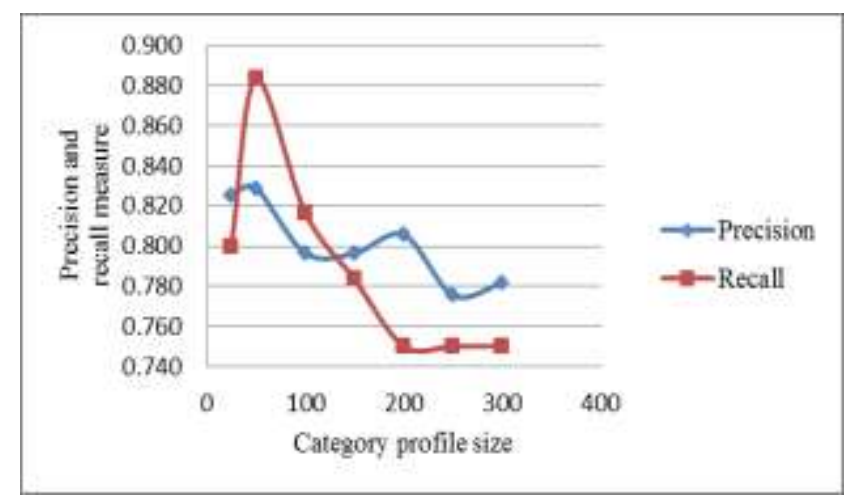

Figure 7. Graphical representation of Precision and Recall for each profile size.

From our main test using 180 articles we have evaluated the performance of each category. The precision and recall measures are shown in table 5 .

Only category DLP and VPN scored perfect precision as all detected documents were relevant. The lowest precision was scored by category Firewall and the score was as low as 0.68 . On the other hand no category scored perfect recall in this experiment as the highest recall measure was 0.967, scored by both Antivirus and Encryption categories. Also, the lowest recall measure was 0.467 and it was scored by category DLP. Lower precision might introduce false positive while lower recall might lead to false negative.

TABLE 5. PRECISION AND RECALL FOR EACH CATEGORY USING 50 $\mathrm{N}$-GRAMS

\begin{tabular}{|c|c|c|}
\hline Category & Precision & Recall \\
\hline Antivirus & 0.744 & 0.967 \\
DLP & 1.000 & 0.467 \\
IDS & 0.788 & 0.867 \\
Encryption & 0.725 & 0.967 \\
Firewall & 0.686 & 0.800 \\
VPN & 1.000 & 0.667 \\
\hline Average & $\mathbf{0 . 8 2 4}$ & $\mathbf{0 . 7 8 9}$ \\
\hline
\end{tabular}


We can improve recall measure for any category by modifying the category profiles. We have used the results already found in the category modification test in subsection $\mathrm{C}$ of section $\mathrm{V}$ to check the effect on precision and recall measures. By using the modified DLP profile, the recall score for the DLP category increased from 0.467 to 0.833 , however the precision score was reduced from 1.0 to 0.925. Also there were some other effects on other categories after the DLP category profile was modified.

TABLE 6. PRECISION AND ReCAll Using MOdifiEd CATEGory PROFILE

\begin{tabular}{|c|c|c|}
\hline Category & Precision & Recall \\
\hline Antivirus & 0.829 & 0.967 \\
DLP & 0.926 & 0.833 \\
IDS & 0.862 & 0.833 \\
Encryption & 0.806 & 0.967 \\
Firewall & 0.727 & 0.800 \\
VPN & 1.000 & 0.667 \\
\hline Average & $\mathbf{0 . 8 5 8}$ & $\mathbf{0 . 8 4 4}$ \\
\hline
\end{tabular}

From table 6 we can see that the precision measure improved for Antivirus, IDS, Encryption and Firewall categories. On the other hand there was no effect on the recall measure for all categories except category IDS, where recall score decreased from 0.867 to 0.833 . Also the overall average improved for both precision and recall after modification. Therefore, these results endorse the idea of profile modification.

\section{F. Limitation of $N$-gram Based Classification}

As mentioned earlier in this paper using N-gram based classification has many advantages over some traditional text analysis methods. However, we came across some limitations in our method which is worth noting. One of the most common problems in data leakage prevention is the use of cryptographic methods to avoid detection. In our case having an encrypted document will definitely impose a great challenge, and it will be impossible to identify the contents of the document without using proper cryptanalysis tools. Moreover, our method showed some efficiency in detecting modified documents, but modifying or replacing the word could lead to wrong classification or misclassification. It is common for people to use word synonyms in documents to avoid measures such as spam filtering and plagiarism detection. This might significantly affect our method making it ineffective.

In addition, our method is based on word-grams and statistical analysis, therefore documents with untraditional sensitive data such as words with special characters, long numbers and non-English characters might not be recognised. The document size is an issue we should consider; from previous analysis we have shown that very big documents can produce large number of $\mathrm{N}$-grams resulting in large distance measures. This will negatively affect the classification process. Also very short documents can impose a challenge to our method, for example if we have a document with few lines the produced $\mathrm{N}$-gram profile will include a small number of category-related $\mathrm{N}$ grams. Therefore a wrong classification is possible.

\section{CONCLUSION AND FUTURE WORK}

In this paper we have studied the effectiveness of using $\mathrm{N}$-gram based classification to categories documents. We have covered most aspects in using $\mathrm{N}$-grams for data classification, such as selecting an optimum size for the category profile and selecting between one-word and twoword N-grams. Moreover, we have tested 180 articles from various sources against six predefined categories. Our preliminary results showed acceptable number of correct classifications as we were able to correctly classify 142 articles out of 180. Moreover, we have improved our overall classification by adding ten common $\mathrm{N}$-grams to the DLP category. The percentage of correct classification increased from $78.8 \%$ to $85 \%$ after modification, and we expect even better results if there were to be more modification to category profiles. In addition, with our limited experiments on document modification, we have achieved high level of correct document classification. We were able to identify 28 documents out of 30 even after various modifications.

Our experiments have shown high levels of recall and precision as we were able to score as high as $100 \%$ for precision and $96.7 \%$ for recall. These results are considered relatively high comparing with other related work. We have also shown that it is possible to improve the average precision and recall measures by using modified category profiles. The average precision increased from 0.824 to 0.858 after modification, also the average recall increased from 0.789 to 0.844 after modification.

Since the results of modifying the DLP category profile were encouraging, as a future direction to this research we propose an automatic category profile update. An automatic update of category profiles would have to include common $\mathrm{X}$-counts addition to the category profile. To achieve this we might need to create a machine learning algorithm that has the ability to select most common $\mathrm{N}$-grams from an $\mathrm{X}$ count database. This database should be exclusive to each category and should have frequency-sorted N-grams from the most common to the least common. Also, we propose an automatic ranking update for the category profile. Instead of using constant category profile, we can regularly update the N-gram ranking by developing another algorithm. This algorithm should have the ability to rank the most common detected N-grams in correctly classified documents. As a result, the more documents we test the more up to date profiles we can have. This will guarantee the inclusion of latest topics within a specific category. 


\section{REFERENCES}

[1] P. Raman, H. G. Kayacık, and A. Somayaji, "Understanding Data Leak Prevention," in 6th Annual Symposium on Information Assurance (ASIA'11), 2011, p. 27.

[2] "Global Data Leakages \& Insider Threats Report: 2011," InfoWatch Analytical Labs2011.

[3] P. Karhula, "What is the effect of WikiLeaks for Freedom of Information?," FAIFE Spotlight [online], vol. 19, 2011.

[4] R. Mogull, "Understanding and Selecting a Data Loss Prevention Solution," SANS Institute2010.

[5] A. Kantor, L. Antebi, Y. Kirsch, and U. Bialik, "METHODS FOR DOCUMENT-TO-TEMPLATE MATCHING FOR DATA-LEAK

PREVENTION," ed: Google Patents, 2009.

[6] X. Shu and D. D. Yao, "Data Leak Detection As a Service: Challenges and Solutions," Virginia Tech2012.

[7] W. B. Cavnar and J. M. Trenkle, "N-gram-based text categorization," Ann Arbor MI, vol. 48113, pp. 161-175, 1994.

[8] G. K. Zipf, Human behavior and the principle of least effort. Massachusetts: Addison Wesley, 1949.

[9] L. Khreisat, "Arabic text classification using Ngram frequency statistics a comparative study," in Conference on Data Mining | DMIN'06 |, 2006, p. 79.

[10] S. Zhou and J. Guan, "Chinese documents classification based on N-grams," Computational Linguistics and Intelligent Text Processing, pp. 3150, 2002.

[11] M. Sokolova, K. El Emam, S. Rose, S. Chowdhury, E. Neri, E. Jonker, and L. Peyton, "Personal health information leak prevention in heterogeneous texts," 2009, pp. 58-69.

[12] E. Stamatatos, "Intrinsic plagiarism detection using character n-gram profiles," threshold, vol. 2, pp. $1,500,2009$.

[13] C. E. Landwehr, C. L. Heitmeyer, and J. McLean, "A security model for military message systems," ACM Transactions on Computer Systems (TOCS), vol. 2, pp. 198-222, 1984.

[14] Fletcher, W. H. (2012). kfNgram (Version 1.3.1). Retrieved from http://kwicfinder.com/kfNgram/kfNgramHelp.html 